\title{
LOCAL SPECTRUM AND SUBHARMONICITY
}

\author{
by B. AUPETIT and D. DRISSI
}

(Received 4th January 1995)

\begin{abstract}
Introducing the technique of subharmonic functions, we prove that the local spectrum $S p_{u(\lambda)}(T)$ is almost constant if $u$ is an analytic family of vectors and if the spectrum of $T$ is thin, a result which is similar to the finite-dimensional situation. We apply this result to improve a former result of C. Foias [7] on generalized scalar operators and results of C. Foiaş and F. - H. Vasilescu [8] on generalized commutators.
\end{abstract}

1991 Mathematics subject classification: 47A11, 47B40

\section{Introduction}

Given two commuting linear bounded operators $T$ and $S$ on a complex Banach space $X$ under which conditions on $T$ and $S$, can we assert the uniqueness property for the local resolvent of $T+S$ ? As shown at the end of this paper, this is the most basic problem in local spectral theory of the commutator $C(T, S)$. This problem was initiated by C. Foias [7] in 1960 who solved it for generalized scalar operators, see also C. Foias \& F. -H. Vasilescu [8], F. -H. Vasilescu $[14,15]$, and K. B. Laursen \& M. M. Neumann [10]. Recently, S. L. Sun [13] has obtained the remarkable result that the sum of two commuting bounded linear operators, both with Dunford property (C) (for the definition see [5]), will have the uniqueness property for the local resolvent.

In Section 1, we recall some preliminary results on local spectral theory. For more details on this topic we refer to the classical monographs of I. Colojoară \& C. Foiaş [4], and F. -H. Vasilescu [15].

In Section 2, we study the variation of $\lambda \rightarrow S p_{u(\lambda)}(T)$ where $u$ is an analytic function defined on a domain $D$ with values in a Banach space $X$. Unfortunately, this multifunction is not upper semicontinuous, even in the finite-dimensional situation. But, using regularization, we are able to prove that this multifunction has nice subharmonic properties (Theorem 2.1) which imply interesting results on the constancy of $S p_{u(\lambda)}(T)$ if the spectrum of $T$ is thin. We finish with a striking application of this subharmonic technique (Theorem 2.5 and Corollary 2.6 ) improving strongly the former result of $\mathrm{C}$. Foiaş on generalized scalar operators [7], and we deduce applications to generalized commutators (Corollaries 2.7, 2.8, 2.9, 2.10).

These partial results suggest that the general theory of analytic multifunctions (see [1]) has an important rôle to play in local spectrum theory. 


\section{Preliminary}

Let $T \in \mathscr{B}(X)$ and $x \in X$. We define $\Omega_{x}$ to be the set of $\alpha \in \mathbb{C}$ for which there exists a neighbourhood $V_{\alpha}$ of $\alpha$ and $u$ analytic on $V_{\alpha}$ with values in $X$ such that $(\lambda-T) u(\lambda)=x$ on $V_{a}$. This set is open and contains the complement of the spectrum of $T$. The function $u$ is called a local resolvent of $T$ at $x$ on $V_{x}$ By definition the local spectrum of $T$ at $x$, denoted by $S p_{x}(T)$, is the complement of $\Omega_{x}$, so it is a compact subset of $S p(T)$.

We denote by $r(T)$ the spectral radius of $T$ which is equal to $\lim _{k \rightarrow \infty}\left\|T^{k}\right\|^{1 / k}$. By convention, for $x \neq 0$,

$$
r_{x}(T)= \begin{cases}\operatorname{Max}\left\{|\alpha|: \alpha \in S p_{x}(T)\right\}, & \text { if } S p_{x}(T) \neq \emptyset \\ 0, & \text { if } S p_{x}(T)=\emptyset\end{cases}
$$

and $\rho_{x}(T)=\lim _{x \rightarrow \infty}\left\|T^{k} x\right\|^{1 / k} \leqq r(T)$. It is well-known that in general $\left\|T^{k} x\right\|^{1 / k}$ has no limit. From the formal identity $(\lambda-T) \frac{1}{\lambda} \sum_{k=0}^{\infty}\left(\frac{T}{\lambda}\right)^{k} x=x$, we conclude that we have $r_{x}(T) \leqq \rho_{x}(T) \leqq r(T)$.

In general the set $S p_{x}(T)$ may be empty even for $x \neq 0$. But for $x \neq 0$, the local spectrum of $T$ is non-empty if $T$ satisfies the uniqueness property for the local resolvent, that is $(\lambda-T) v(\lambda)=0$ implies $v \equiv 0$ for any analytic function $v$ defined on any domain $D$ of $\mathbb{C}$ with values in $X$. N. Dunford [5] noticed easily that $T$ has this property if the spectrum of $T$ has no interior points. So this happens if $T$ has a finite, countable or real spectrum. For the operators satisfying the uniqueness property for the local resolvent there is a unique local resolvent at $x$ which is the analytic extension of $(\lambda-T)^{-1} x$. Using this property and Liouville's Theorem it is easy to conclude that $S p_{x}(T) \neq \emptyset$ for $x \neq 0$. Also in this case the local spectral radius $r_{x}(T)$ is equal to $\rho_{x}(T)=\lim _{k \rightarrow \infty}\left\|T^{k} x\right\|^{1 / k}$. In general this last property is false.

Lemma 1.1. (Holomorphic Functional Calculus for Local Spectrum). Let $T \in \mathscr{B}(X)$, $x \neq 0$, and $f$ holomorphic on a neighbourhood $D$ of $S p(T)$. Then $f\left(S p_{x}(T)\right)$ is included in $S p_{x}(f(T))$. If $f$ is injective on $D$ then $f\left(S p_{x}(T)\right)=S p_{x}(f(T))$. Moreover if $T$ has the uniqueness property for the local resolvent then we have the same property for any $f$ holomorphic.

Proof. This is essentially the proof given in [6, Theorem 1.6, p. 6]. The injective case is obtained by applying the first case to $f^{-1}$ and the operator $f(T)$.

\section{Analytic results}

Usually generalized scalar operators on a Banach space $X$ are defined in term of the spectral distribution (see [4]). But this notion is equivalent to the following one: we say that $N \in \mathscr{B}(X)$ is a generalized scalar operator if $N=H+i K$ where $H, K \in \mathscr{B}(X)$, $H K=K H$ and $H, K$ are in the class $\mathscr{S}$ of real generalized scalar operators defined by the growth condition 


$$
\left\|e^{i t T}\right\| \leqq C(1+|t|)^{n}, t \in \mathbb{R},
$$

for some integer $n \geqq 0$. Such operators of the class $\mathscr{S}$ have a real spectrum, consequently they satisfy the uniqueness property for the local resolvent. In 1960, C. Foias [7] proved that these generalized scalar operators also have the uniqueness property for the local resolvent.

First we shall prove some analytic property of the local spectrum in order to generalize Foiaş's result.

We refer to [1] for all standard definitions and properties concerning subharmonic functions and potential theory.

Let $D$ be a non-empty open subset of $\mathbb{C}$ and $g: D \rightarrow \mathbb{R} \cup\{-\infty\}$ which is locally upper bounded on $D$. The upper regularization $g^{*}$ of $g$ is by definition

$$
g^{*}(\lambda)=\varlimsup_{\mu \rightarrow \lambda} g(\mu) \geqq g(\lambda) .
$$

This function is upper semicontinuous on $D$.

Theorem 2.1. Let $T \in \mathscr{B}(X)$ satisfying the uniqueness property for the local resolvent and let $u$ be an analytic function from an open subset $D$ of $\mathbb{C}$ into $X$. Then $g$ : $\lambda \rightarrow \log r_{u(\lambda)}(T)$ satisfies the mean inequality property (1); consequently $g^{*}$ is subharmonic on $D$. Moreover the set of $\lambda$ such that $g^{*}(\lambda)>g(\lambda)$ is a polar subset of $D$.

Proof. Because $T$ has the uniqueness property for the local resolvent we have $g(\lambda)=\lim _{k \rightarrow \infty} \mathbf{g}_{\mathbf{k}}(\lambda)$ where $g_{k}(\lambda)=\frac{1}{k} \log \left\|T^{k} u(\lambda)\right\|$. For $k \geqq 1$ fixed, the function $\lambda \rightarrow T^{k} u(\lambda)$ is analytic so, by the Gauss formula on a closed disc $\bar{D}\left(\lambda_{0}, r\right)$ included in $D$, we have

which implies

$$
T^{k} u\left(\lambda_{0}\right)=\frac{1}{2 \pi} \int_{0}^{2 \pi} T^{k} u\left(\lambda_{0}+r e^{i \theta}\right) d \theta
$$

$$
\left\|T^{k} u\left(\lambda_{0}\right)\right\| \leqq \frac{1}{2 \pi} \int_{0}^{2 \pi}\left\|T^{k} u\left(\lambda_{0}+r e^{i \theta}\right)\right\| d \theta
$$

This implies that $\lambda \rightarrow\left\|T^{k} u(\lambda)\right\|$ is subharmonic on $D$. Let $\alpha \in \mathbb{C}$, we have $\mid e^{\alpha \lambda}\left\|T^{k} u(\lambda)\right\|$ $=\left\|T^{k} v(\lambda)\right\|$ where $v(\lambda)=e^{\alpha \lambda} u(\lambda)$ is analytic on $D$. So, by the first part of the proof, we have $\left|e^{\alpha \lambda}\right|\left\|T^{k} u(\lambda)\right\|$ subharmonic on $D$. By the Montel-Radó theorem $([1$, p. 176]) this implies that $g_{k}$ is subharmonic and continuous on $D$, so in particular

$$
g_{k}\left(\lambda_{0}\right) \leqq \frac{1}{2 \pi} \int_{0}^{2 \pi} g_{k}\left(\lambda_{0}+r e^{i \theta}\right) d \theta
$$

Consequently 


$$
\begin{aligned}
g\left(\lambda_{0}\right)=\operatorname{Tim}_{k \rightarrow \infty} g_{k}\left(\lambda_{0}\right) & \leqq \frac{1}{2 \pi} \operatorname{Tim}_{k \rightarrow \infty}\left(\int_{0}^{2 \pi} g_{k}\left(\lambda_{0}+r e^{i \theta}\right) d \theta\right) \\
& \leqq \frac{1}{2 \pi} \int_{0}^{2 \pi} \lim _{k \rightarrow \infty} g_{k}\left(\lambda_{0}+r e^{i \theta}\right) d \theta=\frac{1}{2 \pi} \int_{0}^{2 \pi} g\left(\lambda_{0}+r e^{i \theta}\right) d \theta
\end{aligned}
$$

Certainly $g^{*}$ satisfies the same mean inequality and is upper semicontinuous, so it is subharmonic.

Let $h_{N}=\sup _{k \geqq N} g_{k}$, the sequence $\left(h_{N}\right)$ is decreasing and pointwise converging to $g$. By a very famous theorem of $\mathrm{H}$. Cartan on upper enveloppes of subharmonic functions (see $\left[3\right.$, p. 77-78] or [9, Theorem 7.39]) we know that $h_{N}^{*}$ is subharmonic on $D$ and that $E_{N}=\left\{\lambda \in D: h_{N}(\lambda)<h_{N}^{*}(\lambda)\right\}$ is a polar subset of $D$. Obviously the sequence $\left(h_{N}^{*}\right)$ is decreasing and converges to $g^{*}$. It is easy to see that $\left\{\lambda \in D: g^{*}(\lambda)>g(\lambda)\right\} \subset \cup_{N \geqq 1} E_{N}$, the last set being a countable union of polar subsets of $D$ which is also polar, so we get the result.

Unfortunately, the multifunction $\lambda \rightarrow S p_{u(\lambda)}(T)$ is not upper semicontinuous, even in the case of matrices. This is the reason why we introduce the upper semicontinuous regularization of the spectrum by

$$
S p_{u(\lambda)}^{*}(T)=\bigcap_{r>0} \bigcup_{\mu \in D,|\mu-\lambda|<r} S p_{u(\mu)}(T)
$$

It is easy to see that the graph of $\lambda \rightarrow S p_{u(\lambda)}^{*}(T)$ is the closure of the graph of $\lambda \rightarrow S p_{u(\lambda)}(T)$; consequentiy, the radius of $S p_{u(\lambda)}^{*}(T)$ is the regularization of $r_{u(\lambda)}(T)$. Moreover $\lambda \rightarrow$ $S p_{u(\lambda)}^{*}(T)$ is upper semicontinuous.

Let $K$ be a compact subset of the complex plane. We call peripherical part of $K$ the intersection of $K$ with the smallest closed disc centred at 0 containing $K$. This peripherical part contains at least a point and all its points have the same modulus.

Lemma 2.2. Let $T \in \mathscr{B}(X)$ satisfying the uniqueness property for the local resolvent and let $u$ be an analytic function from a domain $D$ of $\mathbb{C}$ into $X$. Suppose there exists $\lambda_{0} \in D$ such that $r_{u(\lambda)}(T) \leqq r_{u\left(\lambda_{0}\right)}(T)$, for all $\lambda \in D$. Then there exists $c>0$ such that $r_{u(\lambda)}^{*}(T)=c$ on $D$ and the peripherical part of $S p_{u(\lambda)}^{*}(T)$ is constant on $D$.

Proof. By Theorem 2.1 we conclude that $r_{u(\lambda)}^{*}(T)$ is subharmonic on $D$; hence by the Maximum Principle it is constant. The rest of the argument paraphrases the proof of Theorem 3.4 .11 in [1] replacing $S p f(\lambda)$ by $S p_{u(\lambda)}^{*}(T), S p(f(\lambda)+a z)$ by $S p_{u(\lambda)}^{*}(T+a)$ and $\rho(g(\lambda))$ by $r_{u(\lambda)}^{*}(T+a)$.

We shall say that a compact subset $K$ of the complex plane is nice if for every $\alpha \in \partial K$ there exists $a \notin K$ such that $K \subset\{z:|z-a| \geqq|\alpha-a|\}$. This condition is automatically verified if $K$ is contained in a smooth curve. 
Theorem 2.3. Let $T \in \mathscr{P}(X)$ and let $u$ be an analytic function from a domain $D$ of $\mathbb{C}$ into $X$. Suppose moreover that $S p(T)$ is nice and without interior points. Then $S p_{u(\lambda)}^{*}(T)$ is constant on $D$.

Proof. If $u$ is identically zero on $D$ the result is obvious. Because $T$ satisfies the uniqueness property for the local resolvent, $S p_{u(\lambda)}(T)$ is non-empty on a subdomain $D^{\prime}$ such that $D \backslash D^{\prime}$ is discrete. So without loss of generality we may suppose that $u$ is never zero on $D$. Let $\lambda_{0} \in D$ be fixed and $\alpha \in S p_{u\left(\lambda_{0}\right)}(T)$. Because $\operatorname{Sp}(T)$ is nice there exists $a \notin S p(T)$ such that $S p_{u\left(\lambda_{0}\right)}(T) \subset S p(T) \subset\{z:|z-a| \geqq|\alpha-a|\}$. We apply Lemma 1.1 to $\frac{1}{2}$, which is holomorphic and injective on a neighbourhood of $S p(T-a)$, so we get

$$
r_{u(\lambda)}\left((T-a)^{-1}\right) \leqq r_{u\left(\lambda_{0}\right)}\left((T-a)^{-1}\right)=\frac{1}{|a-\alpha|} \text {, for } \lambda \in D,
$$

which implies, by Lemma 2.2 , that $\alpha \in S p_{u(\lambda)}^{*}(T)$ for every $\lambda \in D$. So $S p_{u\left(\lambda_{0}\right)}(T) \subset S p_{u(\lambda)}^{*}(T)$, for every $\lambda \in D$ and this implies that $S p_{u(\lambda)}^{*}(T)$ is constant on $D$.

If $K$ is finite it is nice, it has no interior points, so the next result improves the finite-dimensional situation.

Theorem 2.4. Let $T \in \mathscr{B}(X)$ be such that $S p(T)$ is nice, without interior points and let $u$ be an analytic function from a domain $D$ of $\mathbb{C}$ into $X$. Then there exists a compact set $K$ and $a$ polar subset $E$ of $D$ such that $S p_{u(\lambda)}(T)=K$ for $\lambda \in D \backslash E$ and $S p_{u(\lambda)}(T) \subset K$, $S p_{u(\lambda)}(T) \neq K$ for $\lambda \in E$.

Proof. By the previous theorem $S p_{u(\lambda)}^{*}(T)$ is constant on $D$, so denote by $K$ this set and denote by $E$ the subset of $D$ for which $S p_{u(\lambda)}(T) \neq K$. We now prove that $E$ is polar. Let $\lambda_{0} \in E$, then there exists $\alpha \in K \backslash S p_{u(\lambda)}(T)$ and $\rho>0$ such that $\bar{D}(\alpha, \rho)$ is disjoint from $S p_{u(\lambda, 0)}(T)$. We know that $S p(T)$ has no interior points, so the disc $D\left(\alpha, \frac{\rho}{2}\right)$ contains some $s \in(\mathbb{Q}+i \mathbb{Q}) \backslash K$ such that

$$
\operatorname{dist}\left(s, S p_{u(\lambda)}(T)>\operatorname{dist}(s, K)\right.
$$

because otherwise we would have

$$
\frac{\rho}{2}>|\alpha-s| \geqq \operatorname{dist}(s, K)=\operatorname{dist}\left(s, S p_{u(\lambda)}(T)\right) \geqq \frac{\rho}{2}
$$

which is absurd. We now fix such an $s \in(\mathbb{Q}+i \mathbb{Q}) \backslash K$ satisfying (2). Because the spectrum of $T$ has no interior points the only holes of $K$ are those surrounding at least one hole of $S p(T)$. In every component of $\mathbb{C} \backslash S p(T)$ we select a point $\alpha(\alpha=\infty$ for the unbounded component), then by Runge's Theorem (see [12 Theorem 13.6]) we can approximate $1 / \mu-s$ on $K$ by a sequence of rational functions $p_{k}(\mu)$ having only poles on the set of $\alpha$. 
Let $\phi_{k}(\lambda)=\log r_{u(\lambda)}\left(p_{k}(T)\right)=\log \operatorname{Max}\left|p_{k}\left(S p_{u(\lambda)}(T)\right)\right|$ which is well-defined because $(T-\alpha)^{-1}$ exists, for every $\alpha$. Then, by Lemma 1.1 and Theorem $2.1, E_{k}(s)=\left\{\lambda: \phi_{k}(\lambda)<\phi_{k}^{*}(\lambda)\right\}$ is polar, consequently $E(s)=\cup_{k \geqq 1} E_{k}(s)$ is also polar. When $k$ goes to infinity we have

$$
\begin{gathered}
\lim _{k \rightarrow \infty} \phi_{k}\left(\lambda_{0}\right)=-\log \operatorname{dist}\left(s, S p_{u\left(\lambda_{0}\right)}(T)\right)<-\log \operatorname{dist}(s, K) \\
=-\log \operatorname{dist}\left(s, S p_{u\left(\lambda_{0}\right)}^{*}(T)\right)=\lim _{k \rightarrow \infty} \phi_{k}^{*}\left(\lambda_{0}\right) .
\end{gathered}
$$

Consequently, $\lambda_{0} \in E(s)$. This implies that $E \subset \cup E(s)$, for all $s \in(\mathbb{Q}+i \mathbb{Q}) \backslash K$, so $E$ is polat.

Theorem 2.5. Let $T, S \in \mathscr{B}(X)$. Suppose that $T S=S T$, that the spectrum of $T$ is nice and without interior points and that the spectrum of $S$ has no interior points. Then $T+S$ has the uniqueness property for the local resolvent.

Proof. Suppose that $u$ is analytic on a domain $D$ of $\mathbb{C}$ and satisfies $(\lambda-(T+S)) u(\lambda)=$ 0 . Suppose that $u$ is not identically zero on $D$ then, without loss of generality, we can suppose it is never zero on $D$. For $\alpha \in \mathbb{C}$ we have $(\alpha-T) u(\lambda)=(\alpha-\lambda+S) u(\lambda)$, for $\lambda \in D$. Consequently, if $\alpha$ is not in $S p(\lambda-S)=\lambda-S p(S)$, because $T$ and $S$ commute, we have

$$
(\alpha-T)(\alpha-\lambda+S)^{-1} u(\lambda)=u(\lambda) .
$$

For $\lambda \in D$ fixed, it is obvious that $\alpha \rightarrow(\alpha-\lambda+S)^{-1} u(\lambda)$ is analytic on the complement of $S p(S)-\lambda$. This implies that $S p_{u(\lambda)}(T) \subset \lambda-S p(S)$, for all $\lambda \in D$. Taking the regularization we get

$$
S p_{u(\lambda)}^{*}(T) \subset \lambda-S p(S), \text { for all } \lambda \in D .
$$

By Theorem 2.3, $S p_{u(\lambda)}^{*}(T)$ is constant and non-empty on $D$, so let $\alpha$ be a point which is always in $S p_{u(\lambda)}^{*}(T)$, then we have $\lambda-\alpha \in S p(S)$, for all $\lambda \in D$. Hence $S p(S)$ contains the open set $D-\alpha$ and this is a contradiction. So $u \equiv 0$ on $D$ and $T+S$ satisfies the uniqueness property for the local resolvent.

Corollary 2.6. Let $T, S \in \mathscr{B}(X)$. Suppose $T S=S T$ and $S p(T), S p(S)$ real, then $T+i S$ has the uniqueness property for the local resolvent.

In this particular case of operators with real joint spectrum F. -H. Vasilescu [14, Theorem 5.8] has obtained that each $n$-tuples of commuting operators with real joint spectrum possesses the uniqueness property for the local resolvent.

Let $T, S \in \mathscr{B}(X)$, the generalized derivation $C(T, S)$ defined by $T, S$ is by definition the operator defined on $\mathscr{B}(X)$ by $C(T, S) A=T A-A S$. If $T=S$ it is an inner derivation. In [9, Proposition 2.2], C. Foias and F. -H. Vasilescu proved that $C(T, S)$ has the uniqueness property for the local resolvent if $T$ and $S$ are decomposable operators. They applied this fact to obtain two very interesting inclusions for the local spectra (see Corollaries 2.9, 2.10 below). Even at the end of their paper they noticed that the 
uniqueness property for $C(T, S)$ could be obtained supposing only $S$ decomposable and $T$ with the uniqueness property and the property that $\left\{x \in X: S p_{x}(T) \subset F\right\}$ is closed for every closed subset $F$ of $\mathbb{C}$ (Dunford's property). This last result has been extended by K. B. Laursen and M. M. Neumann ([10, Theorem 2.4]) supposing only that $S$ has property $(\delta)$ and $T$ has Dunford's property (for the definition of property $(\delta)$ see this last paper, it is well-known that $S$ decomposable implies that $S$ has property $(\delta)$ ).

It is easy to see that, in general, generalized derivations, even inner derivations, do not have the uniqueness property for the local resolvent. Taking the left shift operator $T$ on $l^{2}$ then, there exists for $0<|\lambda|<1$ an analytic family $x(\lambda)$ of vectors such that $(\lambda-T) x(\lambda)=0$ where

$$
x(\lambda)=\frac{e_{1}}{\lambda}+e_{2}+\lambda e_{3}+\cdots+\lambda^{n-2} e_{n}+\cdots
$$

So denoting by $A(\lambda) \in \mathscr{B}(X)$ the operator defined by $A(\lambda) y=(y \mid x(\lambda)) x(\lambda)$ we obtain an analytic family of operators such that on $l^{2} \oplus l^{2}$ we have

$$
\left(\begin{array}{ll}
T & 0 \\
0 & 0
\end{array}\right)\left(\begin{array}{cc}
0 & A(\lambda) \\
0 & 0
\end{array}\right)-\left(\begin{array}{cc}
0 & A(\lambda) \\
0 & 0
\end{array}\right)\left(\begin{array}{ll}
T & 0 \\
0 & 0
\end{array}\right)=\lambda\left(\begin{array}{cc}
0 & A(\lambda) \\
0 & 0
\end{array}\right)
$$

which imply that the inner derivation defined by $\left(\begin{array}{cc}T & 0 \\ 0 & 0\end{array}\right)$ on $l^{2} \oplus l^{2}$ does not have the uniqueness property for the local resolvent.

We now prove that $C(T, S)$ has the uniqueness property for the local resolvent, if $T$ and $S$ have spectra with good topological properties.

Corollary 2.7. Let $T, S \in \mathscr{B}(X)$. Suppose that the spectrum of one of these operators is nice and without interior points and that the spectrum of the other has no interior points. Then $C(T, S)$ has the uniqueness property for the local resolvent, consequently $S p_{A} C(T, S)$ is non-empty for non-zero $A \in \mathscr{B}(X)$ and there exists a unique local resolvent at $A$.

Proof. We have $C(T, S)=L_{T}-R_{S}$, where $L_{T}$ and $R_{S}$ denote respectively the left multiplication by $T$ and the right multiplication by $S$. These two operators commute; moreover $S p\left(L_{T}\right)=S p(T)$ and $S p\left(R_{S}\right)=S p(S)$. So by Theorem 2.5 applied to $L_{T}$ and $-R_{S}$ we have the result.

Corollary 2.8. Let $T=T_{1}+i T_{2}, S=S_{1}+i S_{2} \in \mathscr{B}(X)$. Suppose that $T_{1} T_{2}=T_{2} T_{1}, S_{1} S_{2}=$ $S_{2} S_{1}$ and that $T_{1}, T_{2}, S_{1}, S_{2}$ have real spectra. Then $C(T, S)$ has the uniqueness property for the local resolvent.

Proof. We have $C(T, S)=L_{T_{1}}-R_{S_{1}}+i\left(L_{T_{2}}-R_{S_{2}}\right)$. Because $L_{T_{1}}, R_{S_{1}}$ commute we have $S p\left(L_{T_{1}}-R_{S_{1}}\right) \subset S p\left(L_{T_{1}}\right)-S p\left(R_{S_{1}}\right) \subset \mathbb{R}$. The same is true for $L_{T_{2}}-R_{S_{2}}$. By hypothesis $L_{T_{1}}-R_{S_{1}}$ and $L_{T_{2}}-R_{S_{2}}$ commute. So we apply Corollary 2.6 . 
This last result can be applied in particular to generalized scalar operators.

Using the arguments of C. Foiaş and F. -H. Vasilescu ([8, Theorem 2.3 and 2.4]) we obtain immediately.

Corollary 2.9. Let $T, S \in \mathscr{B}(X)$ satisfying the hypotheses of Corollary 2.7 or of Corollary 2.8, let $A \in \mathscr{B}(X)$ and let $x \in X$. Then $S p_{A x}(T) \subset S p_{A} C(T, S)+S p_{x}(S)$.

Corollary 2.10. Let $T, S, R \in \mathscr{B}(X)$ satisfying the hypotheses of Corollary 2.7 or of Corollary 2.8 and let $A, B \in \mathscr{B}(X)$. Then $S p_{B A} C(R, T) \subset S p_{A} C(R, S)+S p_{B} C(S, T)$.

All these arguments suggest that parts of the following questions are certainly true:

1. Let $T(\lambda)$ be an analytic family of operators in $\mathscr{B}(X)$, for $\lambda \in D$, having the uniqueness property for the local resolvent and let $u$ be an analytic family of vectors of $X$ defined on $D$, is the multifunction $\lambda \rightarrow S p_{u(\lambda)}^{*}(T(\lambda))$, or a related one, analytic? If this is true Theorems $2.3,2.4,2.5$ can be improved.

2. Is it possible to extend Theorem 2.4 only supposing $S p(T)$ without interior points or even bigger?

3. Is it possible to improve Theorem 2.5 only supposing that $T$ and $S$ commute and their spectra have no interior points?

\section{REFERENCES}

1. B. AUPETIT, A primer on spectral theory (Springer-Verlag, 1991).

2. B. AUPETIT and D. DrIssi, Some spectral inequalities involving generalized scalar operators, Studia Math. 109 (1994), 51-66.

3. M. Brelot, Eléments de la théorie classique du potentiel (Centre de documentation universitaire, Paris, 1965).

4. I. Colojoara and C. Foias, Theory of generalized spectral operators (Gordon and Breach, 1968).

5. N. Dunford, A survey of the theory of spectral operators, Bull. Amer. Math. Soc. 64 (1958), 217-274.

6. I. Erdelyi and R. Lange, Spectral decompositions on Banach spaces (Lecture Notes in Mathematics, Springer-Verlag, 1977).

7.C. Fotas, Une application des distributions vectorielles à la théorie spectrale, Bull. Sci. Math. 84 (1960), 147-158.

8. C. FoIAS and F. -H. VASIlescu, On the spectral theory of commutators, J. Math. Anal. Appl. 31 (1970), 473-486.

9. L. L. Helms, Introduction to potential theory (Robert E. Krieger, 1975).

10. K. B. Laursen and M. M. Neumann, Asymptomatic intertwinning and spectral inclusions on Banach spaces, Czech. Math. J., to appear.

11. H. Radiavi and P. Rosenthal, Invariant subspaces (Springer-Verlag, 1973).

12. W. Rudin, Real and complex analysis (McGraw-Hill, 1974).

13. S. L. Sun, The sum and product of decomposable operators (Chinese), Northeastern Math. J. 5 (1) (1989), 105-117. 
14. F. -H. Vasilescu, Analytic operators and spectral decompositions, Indiana Univ. Math. J. 34 (1985), 705-722.

15. F. -H. Vasilescu, Analytic functional calculus and spectral decomposition (Editura Academiei, Bucharest and D. Reidel Publishing Co., Dordrecht, 1982).

Département de Mathematiques et de Statistique

UNIVERSITE LAVAL

Québec QC

CANADA G1K 7P4

and

Department of Mathematics

Faculty of SCIENCES

KuWAIT UNIVERSITY

P.O. Box 5969 Safat 13060

KuWAIT 